\title{
Myocardial ASL-CMR perfusion imaging with improved sensitivity using GRAPPA
}

\author{
Terrence R Jao ${ }^{1 *}$, Hung P Do², Krishna S Nayak ${ }^{3}$ \\ From 19th Annual SCMR Scientific Sessions \\ Los Angeles, CA, USA. 27-30 January 2016
}

\section{Background}

Myocardial arterial spin labeling (ASL) is a non-contrast CMR technique for measuring myocardial blood flow (MBF), but has relatively low signal-to-physiological noise (PN). Therefore perfusion measurements are made over several averaged pixels within left ventricular (LV) segments and not individual pixels. Shorter image acquisition times using SENSE parallel imaging has been shown to significantly reduce $\mathrm{PN}$ and improve sensitivity to MBF. However, SENSE is unable to reconstruct images at a field of view (FOV) smaller than the object being imaged and requires a large FOV, which leads to fewer number of pixels for averaging in the LV. ${ }^{1}$ In this study, we demonstrate that GRAPPA parallel imaging (rate 1.6), which has no such FOV restrictions, reduces PN even further than SENSE (rate 2), despite having a longer image acquisition window.

\section{Methods}

Six healthy volunteers were scanned using a $3 \mathrm{~T} \mathrm{GE}$ Signa Excite HD scanner with an 8-channel cardiac coil. Myocardial ASL measurements were made at a single mid short axis slice using flow alternating inversion recovery (FAIR) ASL and snap shot balanced SSFP imaging. Images were accelerated using either SENSE with a reduction factor of $2(96 \times 48)$ or GRAPPA with a reduction factor of 1.6 ( $96 \times 60,24$ ACS lines). This corresponded to $153 \mathrm{~ms}$ and $192 \mathrm{~ms}$ imaging windows respectively. The minimum FOV that caused no aliasing was chosen for SENSE imaging while the minimum FOV that causes no aliasing within the left ventricular myocardium was chosen for GRAPPA imaging ${ }^{1}$. 6 breath-held labeled/control image pairs were acquired for each image acceleration scheme. $\mathrm{MBF}, \mathrm{PN}$, and temporal SNR (TSNR $=\mathrm{MBF} / \mathrm{PN})$ were measured within the left ventricular myocardium ROI.

\section{Results}

GRAPPA and SENSE accelerated ASL were acquired at a FOV of $20.1 \pm 2.8 \mathrm{~cm}$ and $26.6 \pm 3.1 \mathrm{~cm}$ with $133.6 \pm$ 35.7 pixels and $67.6 \pm 13.5$ pixels within the segmented LV respectively. Average per-segment MBF and PN measurements across all subjects from GRAPPA and SENSE were $1.97 \pm 0.44 \mathrm{ml} / \mathrm{g} / \mathrm{min}$ and $1.78 \pm 0.73 \mathrm{ml} / \mathrm{g} / \mathrm{min}$ with a corresponding TSNR of 5.44 and 3.26 respectively.

Table 1 Comparison between GRAPPA and SENSE accelerated cardiac ASL

\begin{tabular}{cccccccccc}
\hline \multicolumn{1}{c}{ Global } & \multicolumn{3}{c}{ Per-segment } \\
\hline & FOV $(\mathbf{c m})$ & MBF (ml/g/min) & PN (ml/g/min) & TSNR & N & MBF (ml/g/min) & PN (ml/g/min) & TSNR & N \\
\hline GRAPPA & $20.1 \pm 2.8$ & $1.73 \pm 0.22$ & $0.21 \pm 0.10$ & $11.48 \pm 8.85$ & $532.0 \pm 132.7$ & $1.97 \pm 0.85$ & $0.44 \pm 0.22$ & $5.44 \pm 3.04$ & $133.6 \pm 35.7$ \\
SENSE & $26.6 \pm 3.1$ & $1.75 \pm 0.85$ & $0.60 \pm 0.34$ & $3.41 \pm 1.38$ & $269.6 \pm 43.2$ & $1.78 \pm 1.04$ & $0.73 \pm 0.55$ & $3.26 \pm 3.15$ & $67.6 \pm 13.5$ \\
p-value & 0.0003 & 0.9681 & 0.0289 & 0.0086 & 0.0061 & 0.3230 & 0.0011 & 0.0013 & $2.6 \times 10-12$ \\
\hline
\end{tabular}

Global analysis was performed on the entire left ventricular myocardium. Per-segment analysis divided the myocardium into 6 regional segments. Values are reported as mean \pm standard deviation across all subjects. (FOV) field of view, (MBF) myocardial blood flow, (PN) physiological noise, (TSNR) temporal signal-tonoise, $(N)$ number of pixels within each segment.

Biomedical Engineering, University of Southern California, Los Angeles, CA,

USA

Full list of author information is available at the end of the article 
No significant difference in MBF was found between the two methods ( $\mathrm{p}=0.3230$ ) while PN found in GRAPPA was significantly lower than that found in SENSE ( $\mathrm{p}=$ $0.0011)$. Global and per segment MBF, PN, and TSNR are summarized in table 1.

\section{Conclusions}

We demonstrate that GRAPPA parallel imaging has lower PN and higher TSNR than SENSE parallel imaging while having similar estimated MBF, despite having a longer imaging window. This is because GRAPPA is able to image at a lower FOV, which increases the number of pixel for averaging and decreases partial voluming of the blood pool at the myocardial borders. Further reductions to $\mathrm{PN}$ are expected by reducing the imaging window by combining GRAPPA with partial Fourier reconstruction or with faster imaging acquisition schemes such as EPI.

\section{Authors' details}

${ }^{1}$ Biomedical Engineering, University of Southern California, Los Angeles, CA, USA. ${ }^{2}$ Department of Astronomy and Physics, University of Southern California, Los Angeles, CA, USA. ${ }^{3}$ Electrical Engineering, University of Southern California, Los Angeles, CA, USA.

Published: 27 January 2016

doi:10.1186/1532-429X-18-S1-P100

Cite this article as: Jao et al:: Myocardial ASL-CMR perfusion imaging with improved sensitivity using GRAPPA. Journal of Cardiovascular Magnetic Resonance 2016 18(Suppl 1):P100.
Submit your next manuscript to BioMed Central and take full advantage of:

- Convenient online submission

- Thorough peer review

- No space constraints or color figure charges

- Immediate publication on acceptance

- Inclusion in PubMed, CAS, Scopus and Google Scholar

- Research which is freely available for redistribution

Submit your manuscript at www.biomedcentral.com/submit 